\title{
Correction to: Engineering behaviour of lime- and waste ceramic dust-stabilized expansive soil under continuous leaching
}

\section{Chukwueloka A.U. Okeke ${ }^{1}$ (I)}

Published online: 21 February 2020

(C) Springer-Verlag GmbH Germany, part of Springer Nature 2020

\section{Correction to: Bulletin of Engineering Geology and the}

Environment (2020)

https://doi.org/10.1007/s10064-019-01648-2

The published version of this article unfortunately contained a mistake in Table 2. Incorrect values of maximum dry density $\left(\gamma_{d \max }\right)$ were mistakenly entered in Table 2 . The correct Table 2 is presented here.

Table 2 Mix ratios and physical properties of natural soil and lime-WCD stabilized soils

\begin{tabular}{|c|c|c|c|c|c|c|}
\hline \multirow{2}{*}{$\begin{array}{l}\text { Soil specimen } \\
\text { Mix ratio }\end{array}$} & \multicolumn{4}{|c|}{ Consistency Limits } & \multicolumn{2}{|c|}{ Proctor compaction test } \\
\hline & $L L(\%)$ & $P L(\%)$ & $P I(\%)$ & $L S(\%)$ & $w_{\text {opt }}(\%)$ & $\gamma_{d \max }\left(\mathrm{kN} / \mathrm{m}^{3}\right)$ \\
\hline $100 \% \mathrm{NS}$ & 60.5 & 32.3 & 28.2 & 18.3 & 13.0 & 16.6 \\
\hline $90 \% \mathrm{NS}+10 \% \mathrm{~L}$ & 51 & 47 & 4.0 & 4.1 & 18.0 & 14.4 \\
\hline $95 \% \mathrm{NS}+5 \% \mathrm{~L}$ & 46 & 41.5 & 4.5 & 5.0 & 16.2 & 15.4 \\
\hline $94 \% \mathrm{NS}+4.5 \% \mathrm{~L}+1.5 \% \mathrm{WCD}$ & 53 & 46.5 & 6.5 & 5.7 & 16.0 & 15.6 \\
\hline $95 \% \mathrm{NS}+3.5 \% \mathrm{~L}+1.5 \% \mathrm{WCD}$ & 58 & 50.5 & 7.5 & 6.5 & 14.1 & 16.2 \\
\hline
\end{tabular}

$N S=$ natural soil $L=$ lime $W C D=$ waste ceramic dust $L L=$ liquid limit $P L=$ plastic limit $P I=$ plasticity index $; S=$ linear shrinkage; $w_{\text {opt }}=$ optimum moisture content; $\gamma_{d \max }=$ maximum dry unit weight

The online version of the original article can be found at https://doi.org/ $10.1007 /$ s10064-019-01648-2

Chukwueloka A.U. Okeke

elo_destiny@yahoo.com

1 Department of Civil Engineering, Covenant University, Ota, Ogun State, Nigeria 